\title{
Pediatric supracondylar fractures of the distal humerus
}

\author{
Jacob W. Brubacher · Seth D. Dodds
}

Published online: 10 May 2008

(C) The Author(s) 2008

\begin{abstract}
Supracondylar fractures of the humerus are a common pediatric elbow injury that are historically associated with morbidity due to malunion, neurovascular complications, and compartment syndrome. True anteroposterior and lateral radiographs are essential not only for an accurate diagnosis, but also for creating a treatment plan for these injuries. A staging system (based on the lateral radiograph) for classifying the severity of the fracture helps guide definitive management. Nondisplaced fractures are treated initially with a posterior splint, followed by a longarm casting. Closed reduction and percutaneous pinning is the preferred treatment for displaced or unstable fractures. If there is any question about fracture stability, patients should be seen within 5 days postoperatively for repeat radiographs to ensure that the reduction and pin fixation has been maintained. Understanding the anatomy, radiographic findings, management options, and complications associated with this fracture allow physicians to limit the morbidity associated with this relatively common pediatric injury.
\end{abstract}

Keywords Supracondylar - Elbow fracture - Pediatric . Percutaneous pinning $\cdot$ Closed reduction

\section{Introduction}

Supracondylar fractures are a common elbow injury in children accounting for $16 \%$ of all pediatric fractures [1]

J. W. Brubacher · S. D. Dodds $(\square)$

Department of Orthopaedics and Rehabilitation, Yale University

School of Medicine, 800 Howard Avenue, New Haven, CT 06520, USA

e-mail: seth.dodds@yale.edu and two-thirds of all hospitalizations for pediatric elbow injuries [2]. These are often significant fractures that may be associated with morbidity due to malunion, neurovascular complications, and compartment syndrome. Historically, a majority of these fractures were treated with closed reduction and long arm casting with the elbow in a position of greater than $100^{\circ}$ of flexion. This flexed posture helped maintain the fracture reduction, but lead to problems with vascular compromise and subsequent Volkman's contracture. After a closed reduction, percutaneous pinning maintains fracture reduction without the need for immobilizing the elbow in significant flexion.

\section{Pathoanatomy}

Age is a key factor in the incidence of supracondylar fractures. This is a fracture that occurs more frequently in skeletally immature children than adults. The peak age for supracondylar fractures is between 6 and 7 years of age [3]. At this age, the supracondylar area is undergoing remodeling and is typically thinner with a more slender cortex, predisposing this area to fracture. The typical mechanism is a fall onto an outstretched hand that puts a hyperextension load on the arm. The distal fragment displaces posteriorly in over $95 \%$ of cases [4]. As the elbow is forced into hyperextension, the olecranon serves as a fulcrum and focuses the stress on the distal humerus causing fracture [5]. The rare flexion-type supracondylar fracture is often the result of a fall directly onto a flexed elbow.

The ossification process of the distal humerus occurs in multiple centers and develops at different ages (Fig. 1). As the ossification centers transition from cartilage to bone, the fracture configuration changes. Understanding the general order of ossification provides landmarks for the 
Fig. 1 Understanding the general order and timing of the different ossification centers within the elbow provides landmarks for the physician to define anatomy on radiographs and to guide treatment. With permission from [6]
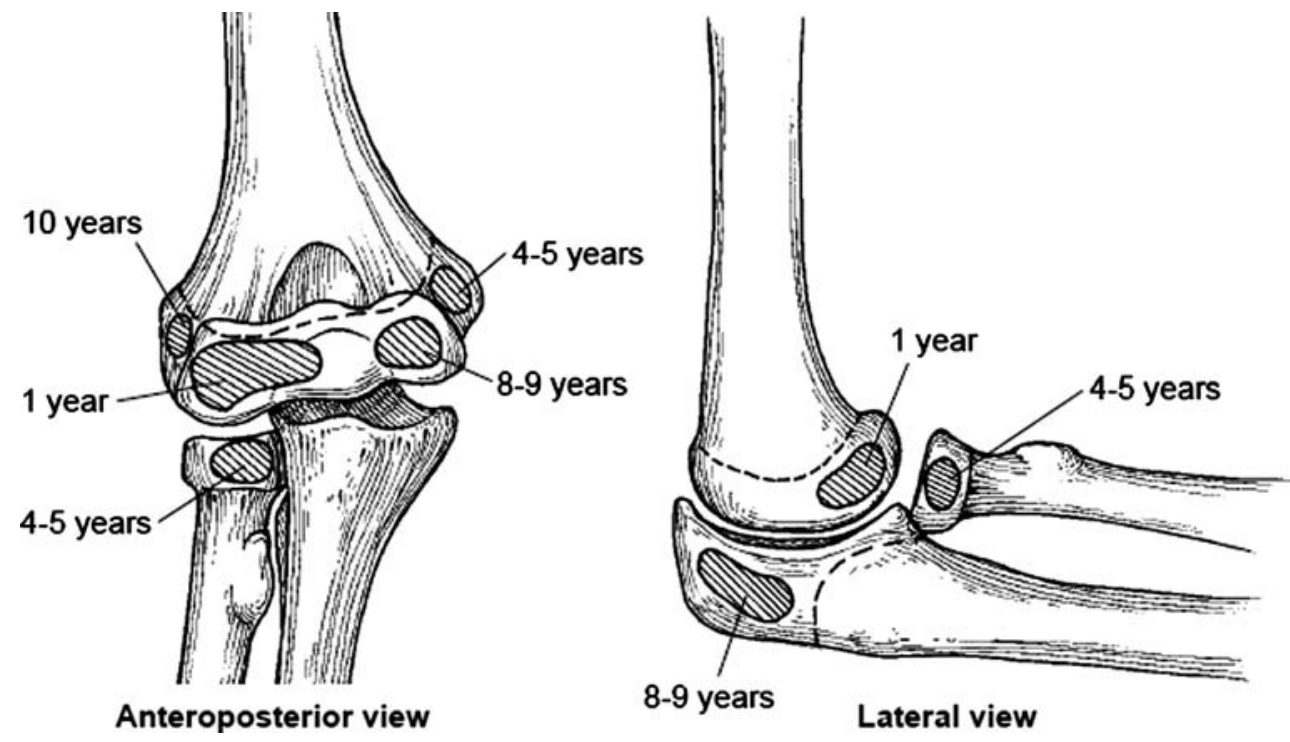

physician to define anatomy on radiographs and to guide treatment. The first to appear is the capitellum at 1 year of age. The radial head and medial epicondyle begin to ossify at 4-5 years of age, followed by the trochlea and olecranon epiphysis at 8 or 9 years of age. The lateral condyle is generally the last to appear at approximately 10 years of age [6].

\section{Physical exam}

A thorough history and physical examination of the extremity is imperative in any significant elbow injury. The initial assessment should rule out any associated trauma. Distal forearm and wrist fractures are not uncommonly associated with this type of elbow injury. The triaging physician should establish the mechanism of injury, all sites of pain or tenderness in the extremity, and the time of onset of this pain. Onset of pain is important to ascertain because muscle ischemia can present as the development of pain in the forearm hours after the injury.

Determining the integrity of the neurovascular structures should be a vital component of the physical exam. Both radial and ulnar pulses must be palpated at the wrist. If no pulse is present, then other signs of perfusion must be checked. For example, the hand should be pink, warm, and demonstrate good capillary refill. The radial, median, and ulnar nerves should each be tested for both motor and sensory function.

If the fracture is completely displaced at the distal humerus, the arm assumes an S-shaped deformity (Fig. 2a). A pucker, dimpling, or ecchymosis of the skin just anterior to the distal humerus may indicate that the proximal, anteriorly directed fragment has penetrated the brachialis muscle and possibly the subcutaneous layer as well
(Fig. 2b). These physical exam findings often indicate that the fracture may be difficult to reduce by simple manipulation alone.

\section{Compartment syndrome}

Increased interstitial pressure within a closed fascial compartment can lead to compartment syndrome. This increased pressure can result in compromised circulation to the nerves and muscles in that compartment. Elevated tissue pressure obstructs venous outflow from the compartment, which further contributes to the increased pressure and swelling. Ischemia occurs once the pressure rises above arteriolar circulation. Muscle and nerve tissue becomes damaged as soon as $4-6 \mathrm{~h}$ after the onset of abnormal pressures.

The first sign of compartment syndrome is disproportionate pain requiring increasing doses of pain medication [7]. Other findings include sensory changes such as paresthesias, loss of active movements in the effected compartment, forearm tenderness, palpable tenseness of the muscles of the forearm (or arm), and pain with passive flexion or extension of the fingers.

It is important to note that distal pulses and capillary refill are not reliable indicators of compartment syndrome. In young children the physical signs of compartment syndrome may be difficult to accurately assess. As a consequence, tissue pressure measurements should be obtained when the diagnosis is uncertain [8].

\section{Diagnostic imaging}

The radiographic study of the injured limb should include an anteroposterior (AP) and a lateral view of the elbow and 

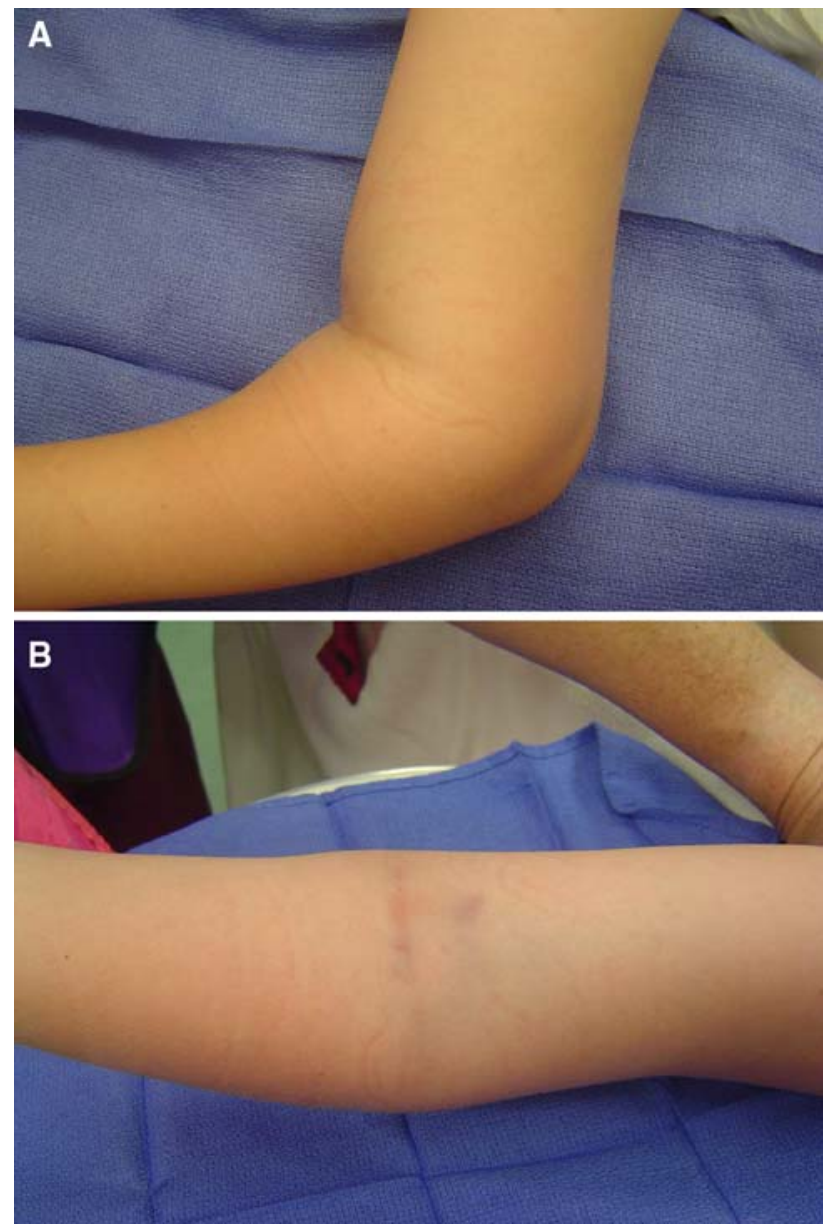

Fig. 2 Clinical appearance of a Type III supracondylar fracture. (a) The lateral view of the elbow demonstrates an S-shaped deformity. (b) The anterior view of the antecubital fossa demonstrates anterior ecchymosis from the anterior spike of the fracture piercing the brachialis muscle

any other sites of deformity, pain, or tenderness. On the AP view, Baumann's angle is commonly used to evaluate fractures as it maintains an estimation of the carrying angle (the varus or valgus attitude of the distal humerus and elbow). This angle is created by the intersection of a line drawn down the axis of the humeral shaft and a line drawn along the growth plate of lateral condyle of the elbow (Fig. 3a). Varus deviation in relation to the proximal humerus produces an increase in Baumann's angle [9]. Radiographs of the contralateral elbow should be used for comparison, if needed, as Baumann's angle varies among all individuals.

On the lateral view, the relationship between the anterior humeral line (a line drawn along the anterior aspect of the humerus) and the ossification center of the capitellum (Fig. 3b) should be examined. In a normal elbow this line should pass through the capitellum. Additionally, on lateral view one may visualize a posterior "fat pad" sign which
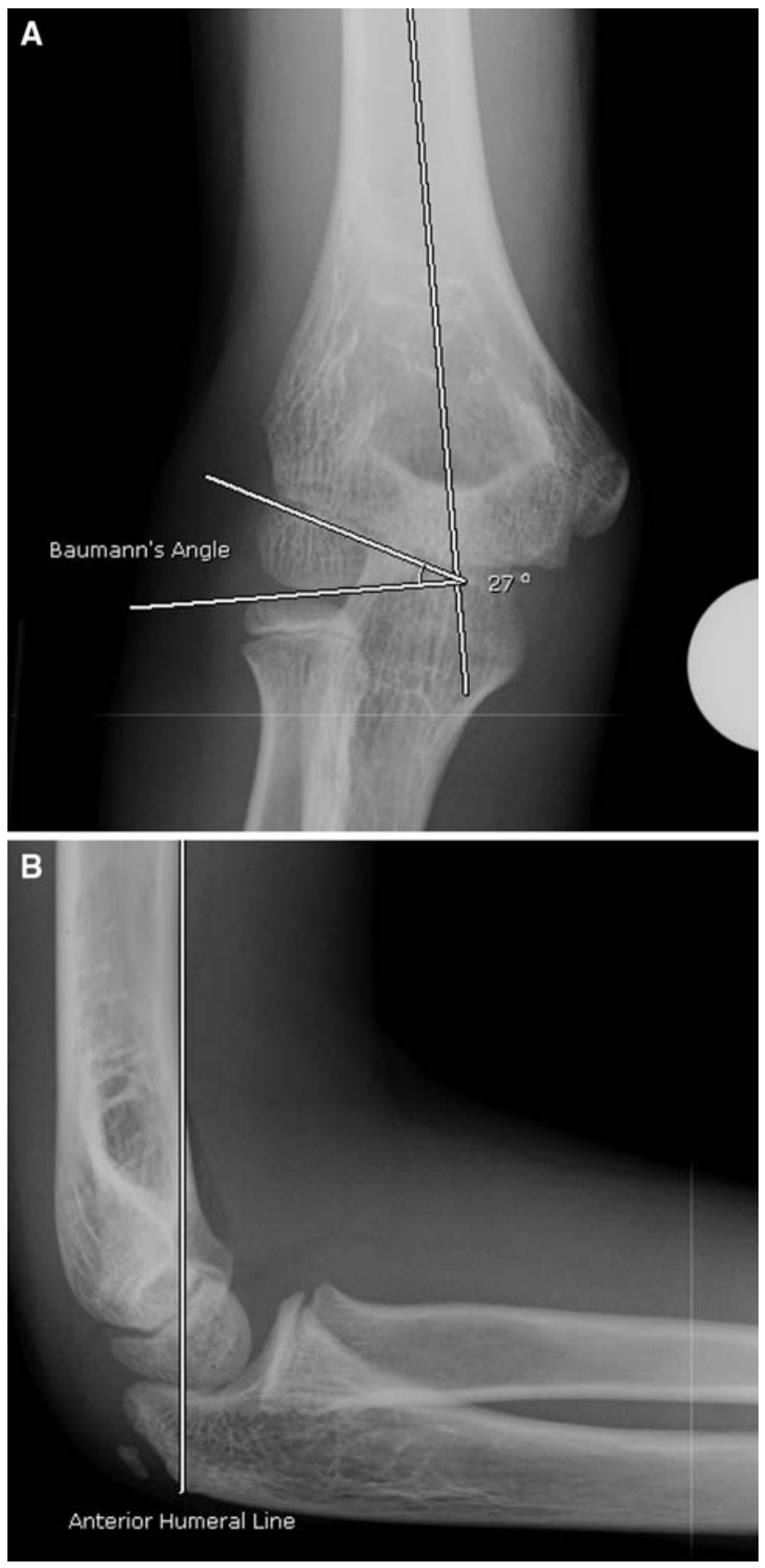

Fig. 3 (a) Baumann's angle is obtained on the anteroposterior radiograph by measuring the angle between a line perpendicular to the longitudinal axis of the humerus and a line parallel to the growth plate of the capitellum. (b) The anterior humeral line is a vertical line drawn directly on the anterior aspect of the distal humeral shaft that should pass through the mid-portion of the capitellum

indicates an intraarticular effusion [10]. An oblique view may be needed to visualize clinically suspected fractures that were not seen on AP or lateral views, especially isolated condyle fractures with intraarticular extension.

Gartland's staging system, based on the lateral radiograph, is widely used for supracondylar fractures as it can help guide treatment [11]. Gartland's Type I fractures are 
nondisplaced. Type II fractures are displaced with angulation, but maintain an intact posterior cortex (Figs. 4a-c). Type III fractures are completely displaced and lack cortical contact (Fig. 5a and b).

\section{Treatment}

After clinical assessment and diagnosis, the elbow should be splinted in a position of comfort (approximately $20^{\circ}-$ $30^{\circ}$ of flexion) to provisionally stabilize the limb. Splinting in full elbow extension is contraindicated because it stretches the neurovascular bundle over the fracture site in displaced or unstable fractures [10]. The application of a comfortable, well padded, and appropriately applied splint is a critical part of the initial management of these injuries, regardless of their definitive treatment.
Nondisplaced (Type I) or minimally displaced fractures in young children can potentially be treated with an aboveelbow cast at $90^{\circ}$ of flexion for 4 weeks. While it is often easiest to visualize displacement or angulation on the lateral radiograph, the Baumann angle on the AP radiograph can be a useful tool to identify and measure varus impaction. When there is varus angulation at the fracture site, strong consideration should be made for closed reduction and percutaneous pinning. More than $10^{\circ}$ of varus malalignment (compared to the contralateral arm) is an indication for operative reduction and pinning [12]. As a general principle, larger diameter pins convey better stability and are more effective at maintaining fracture reduction and alignment.

Angulated fractures that maintain an intact posterior cortex, but have an anterior humeral line that passes anterior to the capitellum on the lateral radiograph (Type
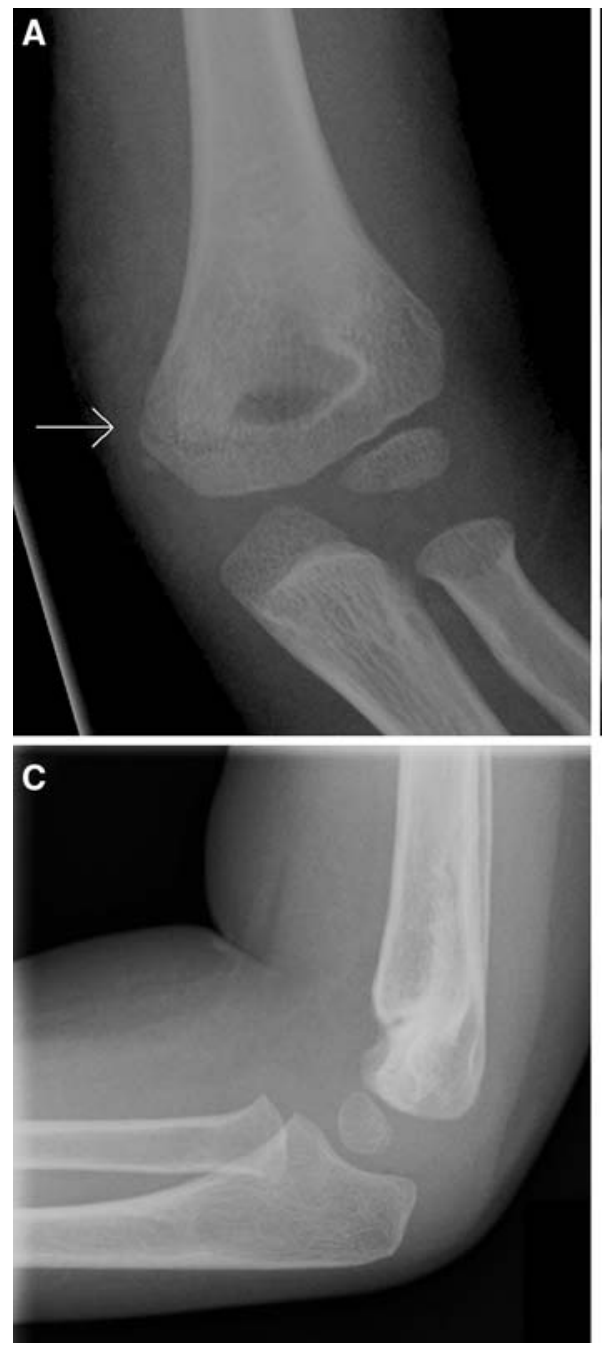

Fig. 4 (a) This anteroposterior radiograph demonstrates a minimally displaced type II supracondylar fracture. (b) The lateral view shows slight posterior angulation of the distal fracture fragment. (c) After

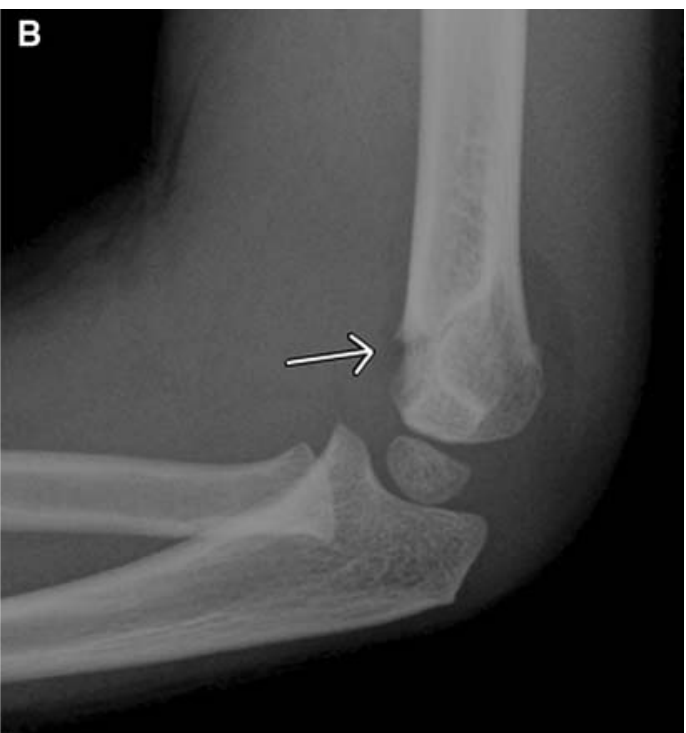

4 weeks in a cast, there has been interval healing and elbow range of motion may be started 

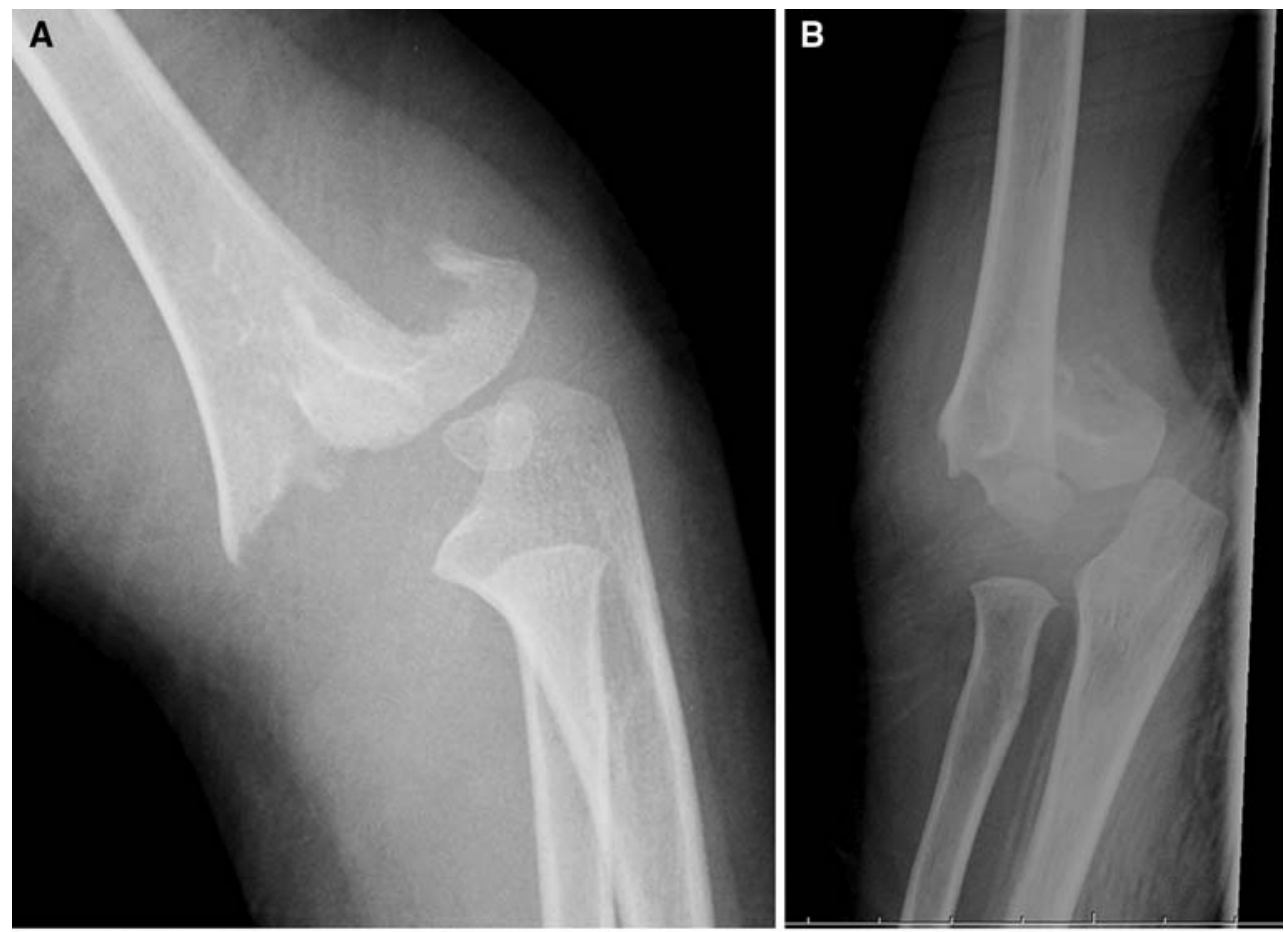

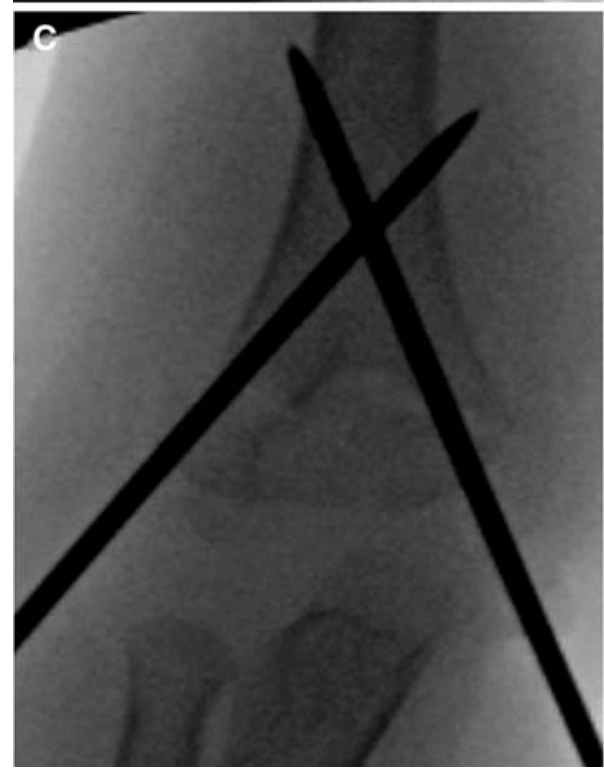

Fig. 5 (a) Anteroposterior and (b) lateral radiographs show complete displacement in this type III supracondylar fracture. (c) Intraoperative anteroposterior radiograph demonstrating fracture reduction and cross

II) require reduction. These may become stable after closed reduction and casting at $90^{\circ}$ of flexion. If more than $90^{\circ}$ of flexion is needed to maintain reduction, then an operative reduction of the fracture with percutaneous pinning should be performed to minimize risks of complications associated with the increased elbow flexion required to maintain reduction in these injuries.

Fractures that create significant displacement of the distal humerus (Type III) are particularly prone to

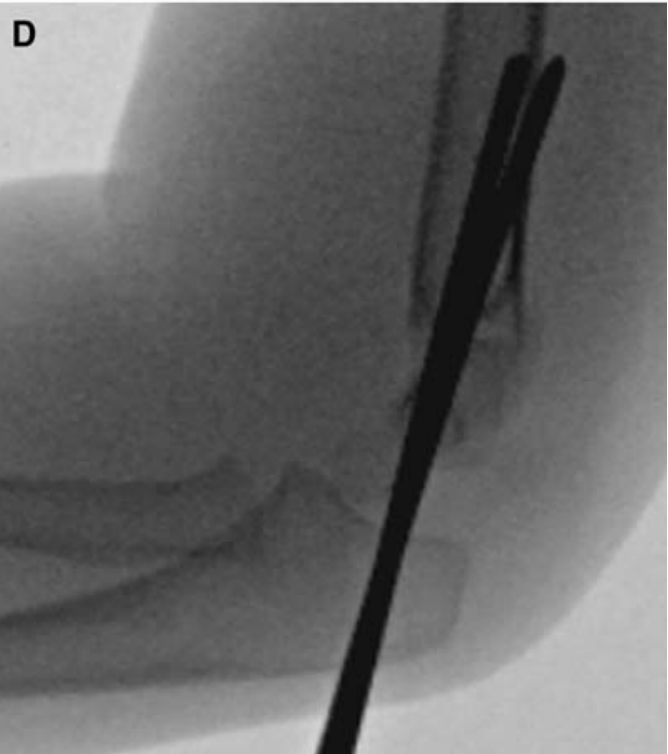

pinning. (d) Lateral view showing restoration of a normal anterior humeral line

neurovascular compromise. Closed reduction and percutaneous pinning is the preferred treatment for displaced fractures (Fig. 5c and d). Fractures with displacement treated by closed reduction and casting have a higher incidence of residual deformity that those managed with operative reduction and pinning [13]. After a careful clinical evaluation that finds no neurovascular injury, an operative fracture may be splinted and managed safely in a delayed fashion (within $24 \mathrm{~h}$ ) while awaiting operative 
fracture reduction. Recent studies have shown that delayed surgical intervention does not increase complication rates [14-16] or the quality of the reduction [17]. However, it is still our feeling that the optimal management of a displaced fracture consists of an operative reduction and percutaneous pinning in an urgent manner and at least within $24 \mathrm{~h}$. Certainly, a child with an operative fracture should be admitted for close observation of the neurovascular status while waiting for operative treatment.

An open reduction is indicated in cases where the fracture is irreducible by closed methods or if the brachial artery has been compromised and requires exploration. Preoperative arterial insufficiency may be improved by operative reduction and pinning, in that a kinked brachial artery, draped over the distal end of the proximal fragment, may become patent after manipulative reduction of the fracture. Lastly, all open supracondylar fractures warrant a surgical debridement of the fracture followed by stabilization.

While postoperative protocols vary from surgeon to surgeon, a typical regiment calls for a long arm, ulnar gutter-type splint or a split long arm cast to control elbow motion and forearm rotation for 4 weeks, followed by pin removal and early range of motion or continued splinting for additional 1-2 weeks. If a stable closed reduction and pinning of the fracture is achieved by an experienced pediatric orthopedic surgeon, follow-up may safely be delayed until the day of pin removal [18]. However, if there is any uncertainty about fracture reduction or stability after pinning, the first follow-up visit should be within 7 days of surgery. This early follow-up for unstable fractures allows for a repeat closed manipulation and pinning if there has been a loss of reduction.

\section{Complications}

The incidence of traumatic and iatrogenic nerve injures with this type of fracture have been recorded as 12-20\% and $2-6 \%$, respectively [19]. The median nerve, specifically the anterior interosseous nerve, (52\%) and radial nerve $(32 \%)$ are most frequently injured in the course of the injury [20]. Most deficits that occur at the time of fracture are neurapraxias (a stretch or contusion of the nerve) and spontaneously recover function in 2-3 months $[21,22]$. If there has been no recovery of function after 46 months, then exploration is indicated. Neurolysis and/or repair have favorable results in children [23]. Iatrogenic nerve deficits often affect the ulnar nerve and result from a pin impinging on the nerve. Management of this complication varies from pin removal and observation to surgical exploration.

Vascular insufficiency resulting from supracondylar fractures has been reported to range from $5 \%$ to $12 \%[24,25]$.
Prompt reduction of the fracture often restores the interrupted arterial flow [25, 26]. After reduction, careful observation and clinical exam are necessary to differentiate between a hand that that is well-perfused with absent pulse from one that is cold, pale, and truly ischemic. Management of a well-perfused hand with an absent pulse varies. In this scenario, many surgeons opt to carefully monitor the child with frequent vascular exams. An arteriogram is often of little use diagnostically as the location of the lesion if often apparent. True vascular insufficiency after reduction calls for surgical exploration.

Angular malunions of the distal humerus are a possible complication of supracondylar fractures. The remodeling potential of the distal humerus is somewhat limited due to the fact that the distal physis contributes only $20 \%$ to the growth of the distal humerus. Remodeling of the angulation in the sagittal plane can occur, but angular deformities in the coronal plane are less likely to remodel-resulting in a cubitus varus or valgus deformity [10].

Cubitus varus, or 'gun-stock deformity', is the most common late complication of this type of fracture. This deformity is the result of fracture malunion and occasionally the partial growth arrest of the medial condylar physis [27]. Proper anatomic reduction and fixation during initial management prevents malunion. Minor varus angulation is generally considered a cosmetic, rather than functional, deformity. A corrective osteotomy may be performed to improve clinically significant malunions.

\section{Summary}

Supracondylar fractures of the humerus are a common pediatric elbow injury that can be associated with neurovascular complications and skeletal deformity. Understanding the anatomy, radiographic findings, complications, and management options associated with this fracture allow physicians to limit the morbidity associated with this injury.

Open Access This article is distributed under the terms of the Creative Commons Attribution Noncommercial License which permits any noncommercial use, distribution, and reproduction in any medium, provided the original author(s) and source are credited.

\section{References}

1. Cheng JC, Shen WY. Limb fracture pattern in different pediatric age groups: a study of 3,350 children. J Orthop Trauma. $1993 ; 7(1): 15-22$

2. Wilkins KE. Fractures and dislocations of the elbow region. In: Rockwood CA Jr, Wilkins KE, King RE, editors. Fractures in children. 3rd ed. Philadelphia: JB Lippincott; 1991. p. 526-617

3. Henrikson B. Supracondylar fracture of the humerus in children. A late review of end-results with special reference to the cause of 
deformity, disability and complications. Acta Chir Scand Suppl. 1966;369:1-72

4. Skaggs D, Pershad J. Pediatric elbow trauma. Pediatr Emerg Care. 1997;13(6):425-34

5. Abraham E, Powers T, Witt P, Ray RD. Experimental hyperextension supracondylar fractures in monkeys. Clin Orthop Relat Res. 1982;(171):309-18

6. Skaggs DL. Elbow fractures in children: Diagnosis and management. J Am Acad Orthop Surg. 1997;5(6):303-12

7. Bae DS, Kadiyala RK, Waters PM. Acute compartment syndrome in children: contemporary diagnosis, treatment, and outcome. J Pediatr Orthop. 2001;21(5):680-8

8. Gulli B, Templeman D. Compartment syndrome of the lower extremity. Orthop Clin North Am. 1994;25(4):677-84

9. Camp J, Ishizue K, Gomez M, Gelberman R, Akeson W. Alteration of Baumann's angle by humeral position: implications for treatment of supracondylar humerus fractures. J Pediatr Orthop. 1993;13(4):521-5

10. Otsuka NY, Kasser JR. Supracondylar fractures of the humerus in children. J Am Acad Orthop Surg. 1997;5(1):19-26

11. Gartland JJ. Management of supracondylar fractures of the humerus in children. Surg Gynecol Obstet. 1959;109(2):145-54

12. De Boeck H, De Smet P, Penders W, De Rydt D. Supracondylar elbow fractures with impaction of the medial condyle in children. J Pediatr Orthop. 1995;15(4):444-8

13. Pirone AM, Graham HK, Krajbich JI. Management of displaced extension-type supracondylar fractures of the humerus in children. J Bone Joint Surg Am. 1988;70(5):641-50

14. Iyengar SR, Hoffinger SA, Townsend DR. Early versus delayed reduction and pinning of type III displaced supracondylar fractures of the humerus in children: a comparative study. J Orthop Trauma. 1999;13(1):51-5

15. Leet AI, Frisancho J, Ebramzadeh E. Delayed treatment of type 3 supracondylar humerus fractures in children. J Pediatr Orthop. 2002;22(2):203-7

16. Mehlman CT, Strub WM, Roy DR, Wall EJ, Crawford AH. The effect of surgical timing on the perioperative complications of treatment of supracondylar humeral fractures in children. J Bone Joint Surg Am. 2001;83-A(3):323-7
17. Carmichael KD, Joyner K. Quality of reduction versus timing of surgical intervention for pediatric supracondylar humerus fractures. Orthopedics. 2006;29(7):628-32

18. Ponce BA, Hedequist DJ, Zurakowski D, Atkinson CC, Waters PM. Complications and timing of follow-up after closed reduction and percutaneous pinning of supracondylar humerus fractures: follow-up after percutaneous pinning of supracondylar humerus fractures. J Pediatr Orthop. 2004;24(6):610-4

19. Ramachandran M, Birch R, Eastwood DM. Clinical outcome of nerve injuries associated with supracondylar fractures of the humerus in children: the experience of a specialist referral centre. J Bone Joint Surg Br. 2006;88(1):90-4

20. Campbell CC, Waters PM, Emans JB, Kasser JR, Millis MB. Neurovascular injury and displacement in type III supracondylar humerus fractures. J Pediatr Orthop. 1995;15(1):47-52

21. Ippolito E, Caterini R, Scola E. Supracondylar fractures of the humerus in children. Analysis at maturity of fifty-three patients treated conservatively. J Bone Joint Surg Am. 1986;68(3):333-44

22. McGraw JJ, Akbarnia BA, Hanel DP, Keppler L, Burdge RE. Neurological complications resulting from supracondylar fractures of the humerus in children. J Pediatr Orthop. 1986;6(6):647-50

23. Amillo S, Mora G. Surgical management of neural injuries associated with elbow fractures in children. J Pediatr Orthop. 1999;19(5):573-7

24. Ottolenghi CE. Prophylaxis of Volkmann's contracture in supracondylar fractures of the elbow in children. Rev Chir Orthop Reparatrice Appar Mot. 1971;57(7):517-25

25. Shaw BA, Kasser JR, Emans JB, Rand FF. Management of vascular injuries in displaced supracondylar humerus fractures without arteriography. J Orthop Trauma. 1990;4(1):25-9

26. Garbuz DS, Leitch K, Wright JG. The treatment of supracondylar fractures in children with an absent radial pulse. J Pediatr Orthop. 1996;16(5):594-6

27. Voss FR, Kasser JR, Trepman E, Simmons E Jr, Hall JE. Uniplanar supracondylar humeral osteotomy with preset Kirschner wires for posttraumatic cubitus varus. J Pediatr Orthop. 1994;14(4):471-8 